\title{
Virtual Reality for Individuals with Occasional Paranoid Thoughts
}

Leonie Ascone

University Medical Center

Hamburg-Eppendorf

I.ascone-michelis@uke.de

Karolin Ney

Universität Hamburg

University Medical Center

Hamburg-Eppendorf

ney.karolin@gmail.com

Fariba Mostajeran

Universität Hamburg

mostajeran@informatik.uni-

hamburg.de

Frank Steinicke

Universität Hamburg

steinicke@informatik.uni-

hamburg.de
Steffen Moritz

University Medical Center

Hamburg-Eppendorf

moritz@uke.de

Jürgen Gallinat

University Medical Center

Hamburg-Eppendorf

j.gallinat@uke.de

\section{Simone Kühn}

University Medical Center

Hamburg-Eppendorf

Max Planck Institute for Human

Development

s.kuehn@uke.de

Permission to make digital or hard copies of part or all of this work for personal or

classroom use is granted without fee provided that copies are not made or distributed for profit or commercial advantage and that copies bear this notice and the full citation on the first page. Copyrights for third-party components of this work must be honored. For all other uses, contact the owner/author(s).

CHI '20 Extended Abstracts, April 25-30, 2020, Honolulu, HI, USA.

(c) 2020 Copyright is held by the author/owner(s).

ACM ISBN 978-1-4503-6819-3/20/04.

DOI: https://doi.org/10.1145/3334480.3382918

\begin{abstract}
Individuals with paranoia often experience a high level of self-criticism and negative emotions. Guided compassionfocused (CF) imagery has shown to be successful in reducing these negative emotions and paranoid thoughts. However, some individuals have difficulties with CF imagery. By enabling a sense of presence, immersive virtual environments can overcome these limitations and induce specific emotional responses to support the development of self-compassionate feelings. In our study, we compared an immersive CF (CF-VR) with a controlled VR condition in a student sample of $\mathrm{N}=21$ participants with slightly elevated symptoms of paranoia. A virtual mission on the moon was designed and implemented to induce self-compassionate feelings with the help of interacting with a space nebula that represented the power of compassion. Our results show that the CF-VR intervention was well accepted and effective in reducing state paranoid thoughts. Worry decreased significantly within the CF-VR group, while self-compassion increased.
\end{abstract}

\section{Author Keywords}

Virtual Reality Therapy; Psychotic Disorders; Paranoia; Self-Compassion. 


\section{CCS Concepts \\ •Human-centered computing $\rightarrow$ Virtual reality; •Applied computing $\rightarrow$ Consumer health;}

\section{Introduction}

Individuals with paranoia exhibit emotional disturbances such as strong fluctuations in anxiety and depressive symptoms along with self-esteem instability and high levels of self-criticism [22, 24, 5, 10]. We need more effective treatments for paranoia, as effect sizes for classical cognitivebehavioral therapy (CBT) interventions are only small to medium $[15,23]$. A recent meta-analysis suggested that interventions focusing on causal mechanisms (causal interventionist approach) bring about larger changes in delusions than classical CBT interventions [15]. As it makes sense to assume an over-activated threat system in paranoia, interventions that target connectedness, kindness, and soothing might be particularly helpful for these individuals.

In compassion-focused (CF) therapy it is hypothesized that almost all psychological disorders can be attributed to a general imbalance of three central emotion regulation systems hosting threat, drive and excitement, and soothing [8]. All these systems serve important functions: the threat system identifies dangers and initiates according stress responses, the drive and excitement system regulates approach behavior towards rewarding stimuli and activities (including social rank competition), and the soothing system promotes bonding and feelings of (social) safeness, warmth and connectedness. In most psychopathologies, the soothing system seems to be hypo-functional, hypothetically often due to lack of secure attachment relationships or even trauma in important developmental phases. Hence CF therapy aims at strengthening this system using different therapeutic strategies, such as developing sympathy and kindness towards oneself - especially in the face of suffering or failure. Among many other techniques, it draws upon meditation practices and imagery to evoke compassion. According to Gilbert [9] compassion is part of a caring, nurturing and sharing social mentality directed both at the self and others, and this mentality seems to be underdeveloped in paranoid individuals. Therefore, the practice of compassion-focused imagery (CFI), such as imagining a caring and nurturing being conveying compassion towards the self, might be very useful for this patient group. However, previous studies of guided CFI in patients affected by psychosis $[14,1]$ have shown that some individuals had difficulties with the imagery. For example, undesirable and even scary images can appear during treatment (e.g. one patient generated a 'ugly, repulsive, super-human'[14]). Nevertheless, in sub-clinical populations, CFI has been shown to reduce paranoid ideation and negative emotions [12].

Virtual reality (VR) might help to overcome limitations of guided imagery by providing controlled virtual environments that support the development of compassionate feelings while avoiding adverse patient imagery. VR interventions for psychotherapy are known to have several beneficial effects. For instance, VR exposure therapy (ET) has comparable effects as in vivo ET for anxiety disorders [19]. Much less is known about the beneficial effects of VR in the treatment of psychosis. A first pilot trial showed that VR can reduce delusion conviction and anxiety in real-life social situations with large effects $[18,4]$. Besides the reduction of negative affect via exposure, it seems to be possible to evoke positive, warm feelings using VR. A single session pilot trial in depressed subjects showed that receiving compassionate gestures generated by the adult-self towards a child-self lead to significant reductions in depression severity and 


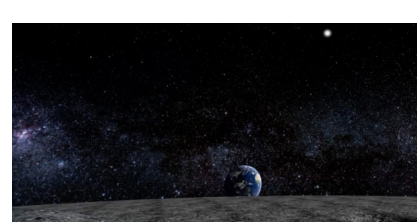

Figure 1: The view of earth

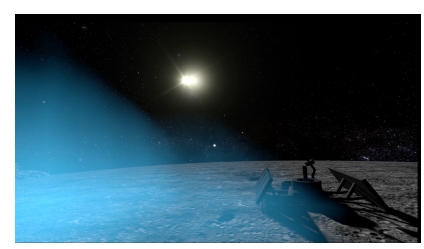

Figure 2: Blue colored nebula appearing on the moon

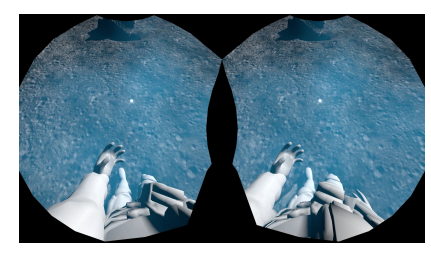

Figure 3: The participants' virtual body

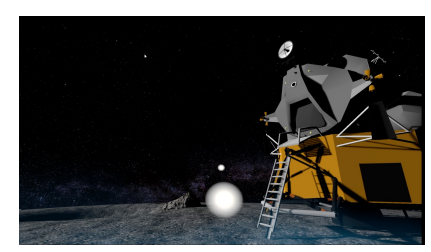

Figure 4: The Apollo 11 lunar module self-criticism, as well as to a significant increase in selfcompassion from baseline to 4-week follow-up [2].

For our intervention, we loosely drew upon a CFI exercise (compassionate colors) from the Compassion-Focused Therapy book for practitioners [8]. As a background scenario we chose space, because it can evoke an overview effect (putting one's existence into a larger perspective), and it elicits feelings of awe, connectedness with humanity, and self-transcendence [25]. The latter phenomena have a striking conceptual overlap with Neff's definition of selfcompassion $[17,16]$, which involves three major components: common-humanity (one's own destiny and suffering being part of the broader human experience), mindfulness (vs. over-identification with problems), and kindness (vs. being judgmental). Hence, we combined a space scenario, where individuals were sent by a mission control (audio via headphones) to the moon (see Figure 1) in order to explore a mysterious nebula (Figure 2) that harbors the power of compassion for oneself and humankind (CF-VR). Participants were gradually guided into opening for feelings of kindness, warmth, wisdom, courage, and strength, while also interacting with light-points within the nebula that intensified their glow by touch (symbolizing the increasing power of compassion). The exact same scenario but with a neutral audio, that instead focused on objective qualities of the nebula and the environment (similar to mindfulness practice), was used as control condition. Both audios and scenarios were about 10 minutes long, and the pausing and pace of the evolvement of the nebula on the moon, alongside with the according instructions, were equal for both conditions.

The present study aimed at piloting the CF-VR vs. control VR in a student sample of $N=21$ participants with slightly elevated symptom levels of paranoia. Those were individuals who indicated to experience paranoid thoughts at least once per month on the frequency scale of the paranoia checklist [7]. Our main outcomes for this pilot trial were paranoia and self-compassion. Furthermore, we examined changes in emotions (both positive and negative).

\section{Methods and Materials}

The Space-Compassion VR application

In an effort to make the space experience more vivid, the space environment was created as an animated game object with rotating planets (with clouds), day/nighttime animations of the planet earth, and the milky way background. The user was placed on the moon surface, close to the Apollo 11 (see Figure 4) lunar module (taken from the official NASA website) to create the impression of being able to take refuge or leave the moon, and still being connected to other people. The VR experience was individualized for each participant by asking them to choose a color they associate with wisdom, warmth, and non-judgement qualities to be used as the color of the self-compassion nebula. To make the nebula interactive, the users were able to manipulate the intensity of its glowing particles. To create a sense of embodiment and agency, the participants were given a virtual body (an astronaut's suit, see Figure 3) which was synchronized with that of the user using an HTC Vive Pro VR head-mounted display (HMD), two controllers (for the hands), and two trackers (for the feet). The SpaceCompassion VR application was developed with the Unity 3D Engine version 2018.3.11f1.

\section{Procedure}

This study was approved by the local Psychological Ethics Committee of the Center for Psycho-social Medicine at the University Medical Center Hamburg-Eppendorf (UKE). The study was performed in the VR Lab at the UKE. The recruitment was accomplished by means of mailing lists, university boards, and online advertisements. Prior to the ex- 
periment, all participants underwent an online screening (T0) to check the eligibility criteria (i.e. at least one paranoid thought per month as measured with the paranoia checklist [7]. Furthermore, demographic data was assessed, including age, sex, employment status, education, history of mental disorders, central nervous medication, and visual impairment (the latter lead to exclusion). Current or past mental disorder was no exclusion criterion. A minimum age of 18 was required, but there was no upper age limit. About one week after the online screening, the laboratory assessment took place. During the laboratory session, all outcomes (paranoia, self-compassion, emotions) were assessed three times: (T1) first directly at the beginning of the session, (T2) after participants were randomized and received a psycho-educative video, depending upon their assignment this was either a neutral video about space (e.g., what is a nebula?) or a video introducing the concept of compassion (e.g., why compassion is important for mental health?), and (T3) third after the VR exposure (CF-VR vs. control VR). At the end of the intervention, we asked participants to rate their perceived personal benefit from the intervention.

\section{Participants}

Mean age within the CF-VR group ( $n=12$ ) was 28.6 years $(S D=13.7)$. Forty-two percent were female, average years of school was $12.2(S D=0.83)$, speaking for a high school education according to German standards. In the control VR group ( $n=9$ ), mean age was $22.1(S D=2.1$ ) and $56 \%$ were female. The mean of school years was 12.1 $(S D=0.33)$. There were no significant differences between the groups in any of these demographic variables $(p>.05)$. In total four participants reported a diagnosed current or past mental disorder. Three of them were in the CF-VR group (one with two past diagnoses: depression and eating disorder; one with past post-traumatic stress disor- der; one with past mental disorder - not specified and current media addiction). One was in the control VR condition (past anxiety and eating disorder).

\section{Questionnaires}

Paranoia

We used a brief version of the German paranoia checklist with three items that has been found to be highly change sensitive [20] (items: 'I need to be on my guard against others', 'People deliberately try to irritate me', 'Strangers and friends look at me critically'), rated on three dimensions: 1) applicability/ predominance of this thought at the moment [0 = 'completely disagree', 11 = 'fully agree'], 2) distress [0 = 'doesn't bother me', 11 = 'bothers me a lot'], and conviction [0='not convinced', 11 = 'completely convinced']. Cronbach's alpha was good to excellent (applicability: $\alpha=.85$; distress: $\alpha=.85$; conviction: $\alpha=.90$ ).

\section{Self-compassion}

We used a brief experimental version of the original SelfCompassion Scale (SCS) [16] as used by Ascone and colleagues [1]. The original SCS measures self-compassionate feelings on three dimensions: self-kindness, common humanity, and mindfulness. The brief version contains one item from each of the subscales (i.e., self-kindness: 'I'm tolerant of my own flaws and inadequacies', common humanity: 'I try to see my failings as part of the human condition', and mindfulness: 'When l'm feeling down I try to approach my feelings with curiosity and openness'). Internal consistency (Cronbach's alpha) was acceptable $(\alpha=.64)$.

Emotions

In order to assess different types of emotions, we used the state part of the State Trait Anxiety Depression Inventory (STADI) [11], which contains 20 items that address feelings, thoughts, and physical states related to nervousness, worry, euthymia and dysthymia (5 items each). Participants are 




Figure 5: Paranoid symptoms



Figure 6: Self-compassion

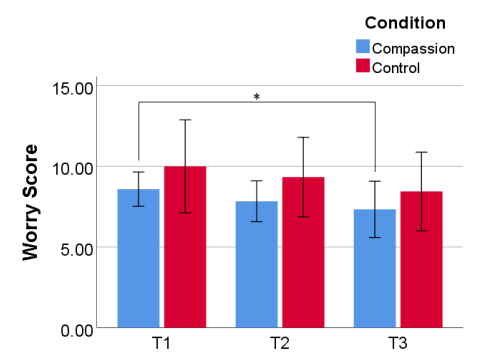

Figure 7: Worry to rate for each item how strongly this applies 'at the moment' on a four-point Likert scale (1 = 'does not apply', 4 = 'fully applies'). Cronbach's alpha was acceptable to good for all subscales (nervousness: $\alpha=.85$; worry: $\alpha=.80$; euthymia: $\alpha=.88$; dysthymia: $\alpha=.73$ ).

Self-rated benefit: psycho-education and VR intervention We assessed the personal benefit individuals experienced during the intervention using a 5-star-rating schema (1 star $=$ rather not helpful; 5 stars = very helpful). The same rating format was used to assess the perceived benefit from the psycho-education (about compassion/ space, depending on the group assignment).

\section{Statistical Analyses}

As assumptions for parametric testing were violated, we used non-parametric within-group difference analyses applying the Friedmann test, in order to analyze overall changes over time within the CF-VR or control VR group. A significant omnibus test was followed up by Bonferroni-adjusted exploratory post-hoc pairwise comparisons between the different assessment points (T1, T2, T3). To compare perceived personal benefit ratings for both conditions we computed the non-parametric Mann-Whitney-U-Test, with a two tailed significance level of .05.

\section{Results}

Paranoia

The following pattern of results (see Figure 5) was identified within the CF-VR group. The applicability/ predominance of paranoid thoughts significantly decreased within the CFVR group $\left(\chi^{2}=14.06, p<.001\right)$. Looking at pair-wise post-hoc comparisons for all three laboratory assessment time-points, this main effect was attributable to a significant change from T1 to T3 ( $Z=-3.06, p=.007)$, suggesting that only a combination of psycho-education and CF-VR related to a significant symptom change. No other pairwisetests reached significance $(p>.05)$ There were no significant effects for paranoia distress $\left(\chi^{2}=0.28, p=.871\right)$ or conviction $\left(\chi^{2}=2.14, p=.343\right)$. There were no significant effects within the control VR group (paranoia - applicability/ predominance: $\chi^{2}=2.38, p=.305$; paranoia-distress: $\chi^{2}=4.00, p=.135$; paranoia - conviction: $\chi^{2}=1.37$, $p=.504)$.

\section{Self-compassion}

There was a significant increase in self-compassion (see Figure 6) $\chi^{2}=16.93(p<.001)$. Post-hoc analyses revealed that the increase took place between T1 and T3 $(Z=3.89, p<.001)$, while there were no significant changes between any other time-points $(p>.05)$, again suggesting that a combination of psycho-education and the CF-VR drove the effect. There was no significant effect for self-compassion in the control VR group $\left(\chi^{2}=3.16, p=\right.$ $.206)$

\section{Emotions}

There was no significant effect within the CF-VR group concerning nervousness $\left(\chi^{2}=5.44, p=.066\right)$. For worry (see Figure 7), a significant result was obtained $\left(\chi^{2}=6.70\right.$, $p=.035)$, which was attributable to changes from T1 to T3 $(Z=2.45, p=.043)$. No other differences between time points were identified for worry. The variable euthymia did not change significantly $\left(\chi^{2}=2.67, p=.264\right)$, nor did dysthymia $\left(\chi^{2}=1.27, p=.529\right)$. Within the control condition, there were no significant effects on any of the emotion subscales (nervousness: $\chi^{2}=3.92, p=.141$; worry: $\chi^{2}=1.50, p=.472$; euthymia: $\chi^{2}=0.83, p=.661$; dysthymia: $\left.\chi^{2}=1.40, p=.497\right)$.

Self-rated benefit: psycho-education and VR intervention Overall, the ratings indicated medium to high perceived personal benefit from the psycho-education (CF: $M d n=4.00$, 
$S D=1.00$; control: $M d n=3.00, S D=0.88)$ and VR intervention (CF-VR: $M d n=3.50, S D=0.78$; control VR: $M d n=4.00, S D=0.97$ ). There were no significant group differences (perceived benefit psycho-education: $U=33.0$, $p=.119$; perceived benefit intervention: $U=48.0$, $p=.650)$.

\section{Discussion}

In sum, the CF-VR (including the psycho-educative part) was associated with changes in state paranoia (decrease), self-compassion (increase), and worry (decrease), while no changes were identified within the control VR. However, all observed changes also included the psycho-educative part on self-compassion, speaking for the importance of explaining the rationale for the intervention to participants in order to evoke significant change. The absence of effects on the paranoia distress and conviction subscales raises the question of clinical relevance of the observed decreases in state paranoia. There are, in our view, two possible explanations for this. First, as our sample was only sub-symptomatic, it is possible that there were bottom effects. Second, it is also possible that distress and conviction are harder to tackle, and that repeated training may be necessary to evoke according change of the affective quality of paranoid thoughts. The effect on self-compassion shows that our intervention indeed alters the target mechanism. The decrease in worry appears promising, as research suggests heightened levels of anxiety and perpetuating worry as important pathogenic mechanisms for paranoia (e.g. [3, 6, 21]). Future analyses should focus on potential mediation effects in order to infer conclusions about mechanisms of change. Of interest, both interventions including psycho-education were rated as at least moderately helpful, speaking for good acceptance.

A clear limitation of our study is the small sample size, which is why we refrained from parametric tests. Replica- tion of the findings in a larger sample, while contrasting both groups (i.e. repeated measures ANOVA) is a necessary next step. Representativeness of the sample is also questionable, as it mainly consisted of highly educated, young individuals (students). Nonetheless, it is known that student populations are often more delusion-prone than the general population [13] and are in the typical age-range for a first manifestation of psychosis. Inclusion of the standard $\mathrm{CFI}$ and a wait-list as additional control conditions in order to examine the advantages of CF-VR remains a topic for further research. In addition, standardized psychiatric diagnostics should be included in future trials.

Based on the qualitative data from our study, the intervention was well accepted and the topic was deemed relevant: "I liked the fact that through the course of the study I learned something about myself and human behavior in general and that it conveyed a positive view upon myself.". However, the VR experience could be improved by including more interactions and more customization (e.g., choice of the guide's voice, color attributes, etc.).

We conclude that our novel space compassion-focused VR has potential to ameliorate paranoid symptoms and worry, and improve compassion. Repeated training in the VR (vs. the standard CFI and/or control VR), larger samples, and replication studies in patients with persecutory delusions appear to be reasonable and promising next steps.

\section{Acknowledgements}

This work was funded by the European Union as well as the German Research Foundation (DFG), the German Federal Ministry of Education and Research (BMBF), and the Federal Ministry for Economic Affairs and Energy (BMWi). 


\section{REFERENCES}

[1] Leonie Ascone, Johanna Sundag, Björn Schlier, and Tania M Lincoln. 2017. Feasibility and effects of a brief compassion-focused imagery intervention in psychotic patients with paranoid ideation: A randomized experimental pilot study. Clinical Psychology \& Psychotherapy 24, 2 (2017), 348-358.

[2] Caroline J Falconer, Aitor Rovira, John A King, Paul Gilbert, Angus Antley, Pasco Fearon, Neil Ralph, Mel Slater, and Chris R Brewin. 2016. Embodying self-compassion within virtual reality and its effects on patients with depression. BJPsych Open 2, 1 (2016), 74-80.

[3] Chloe Foster, Helen Startup, Laura Potts, and Daniel Freeman. 2010. A randomised controlled trial of a worry intervention for individuals with persistent persecutory delusions. Journal of Behavior Therapy and Experimental Psychiatry 41, 1 (2010), 45-51.

[4] Daniel Freeman, Jonathan Bradley, Angus Antley, Emilie Bourke, Natalie DeWeever, Nicole Evans, Emma Černis, Bryony Sheaves, Felicity Waite, Graham Dunn, and others. 2016. Virtual reality in the treatment of persecutory delusions: randomised controlled experimental study testing how to reduce delusional conviction. British Journal of Psychiatry 209, 1 (2016), 62-67.

[5] Daniel Freeman, Graham Dunn, David Fowler, Paul Bebbington, Elizabeth Kuipers, Richard Emsley, Suzanne Jolley, and Philippa Garety. 2012. Current paranoid thinking in patients with delusions: the presence of cognitive-affective biases. Schizophrenia bulletin 39, 6 (2012), 1281-1287.

[6] Daniel Freeman, Graham Dunn, Helen Startup, Katherine Pugh, Jacinta Cordwell, Helen Mander,
Emma Černis, Gail Wingham, Katherine Shirvell, and David Kingdon. 2015. Effects of cognitive behaviour therapy for worry on persecutory delusions in patients with psychosis (WIT): a parallel, single-blind,

randomised controlled trial with a mediation analysis. The Lancet Psychiatry 2, 4 (2015), 305-313.

[7] Daniel Freeman, Philippa A Garety, Paul E

Bebbington, Benjamin Smith, Rebecca Rollinson, David Fowler, Elizabeth Kuipers, Katarzyna Ray, and Graham Dunn. 2005. Psychological investigation of the structure of paranoia in a non-clinical population. The British Journal of Psychiatry 186, 5 (2005), 427-435.

[8] Paul Gilbert. 2010. Compassion focused therapy: Distinctive features. Routledge.

[9] Paul Gilbert. 2014. The origins and nature of compassion focused therapy. British Journal of Clinical Psychology 53, 1 (2014), 6-41.

[10] Paul Hutton, James Kelly, lan Lowens, Peter J Taylor, and Sara Tai. 2013. Self-attacking and self-reassurance in persecutory delusions: A comparison of healthy, depressed and paranoid individuals. Psychiatry Research 205, 1-2 (2013), 127-136.

[11] Lothar Laux, Michael Hock, Ralf Bergner-Köther, Volker Hodapp, and Karl-Heinz Renner. 2013. Das State-Trait-Angst-Depressions-Inventar: STADI; Manual. Hogrefe, Göttingen.

[12] Tania M Lincoln, Felicitas Hohenhaus, and Maike Hartmann. 2013. Can paranoid thoughts be reduced by targeting negative emotions and self-esteem? An experimental investigation of a brief compassion-focused intervention. Cognitive Therapy and Research 37, 2 (2013), 390-402. 
[13] Tania M Lincoln and Eva Keller. 2008. Delusions and hallucinations in students compared to the general population. Psychology and Psychotherapy: Theory, Research and Practice 81, 3 (2008), 231-235.

[14] Sophie L Mayhew and Paul Gilbert. 2008. Compassionate mind training with people who hear malevolent voices: A case series report. Clinical Psychology \& Psychotherapy 15, 2 (2008), 113-138.

[15] Stephanie Mehl, Dirk Werner, and Tania M Lincoln. 2015. Does Cognitive Behavior Therapy for psychosis (CBTp) show a sustainable effect on delusions? A meta-analysis. Frontiers in Psychology 6 (2015), 1450.

[16] Kristin D Neff. 2003. The development and validation of a scale to measure self-compassion. Self and Identity 2, 3 (2003), 223-250.

[17] Kristin D Neff. 2011. Self-compassion, self-esteem, and well-being. Social and Personality Psychology Compass 5, 1 (2011), 1-12.

[18] Roos MCA Pot-Kolder, Chris NW Geraets, Wim Veling Marije van Beilen, Anton BP Staring, Harm J Gijsman, Philippe AEG Delespaul, and Mark van der Gaag. 2018. Virtual-reality-based cognitive behavioural therapy versus waiting list control for paranoid ideation and social avoidance in patients with psychotic disorders: a single-blind randomised controlled trial. The Lancet Psychiatry 5, 3 (2018), 217-226.

[19] Mark B Powers and Paul MG Emmelkamp. 2008. Virtual reality exposure therapy for anxiety disorders: A meta-analysis. Journal of Anxiety Disorders 22, 3 (2008), 561-569.

[20] Björn Schlier, Steffen Moritz, and Tania M Lincoln. 2016. Measuring fluctuations in paranoia: Validity and psychometric properties of brief state versions of the Paranoia Checklist. Psychiatry Research 241 (2016), 323-332.

[21] Xiaoqi Sun, Suzanne H So, Raymond CK Chan, Chui-De Chiu, and Patrick WL Leung. 2019. Worry and metacognitions as predictors of the development of anxiety and paranoia. Scientific Reports 9, 1 (2019), $1-10$.

[22] Viviane Thewissen, Richard P Bentall, Margreet Oorschot, Joost à Campo, Thom van Lierop, Jim van Os, and Inez Myin-Germeys. 2011. Emotions, self-esteem, and paranoid episodes: An experience sampling study. British Journal of Clinical Psychology 50, 2 (2011), 178-195.

[23] Mark van der Gaag, Lucia R Valmaggia, and Filip Smit. 2014. The effects of individually tailored formulation-based cognitive behavioural therapy in auditory hallucinations and delusions: a meta-analysis. Schizophrenia Research 156, 1 (2014), 30-37.

[24] Stefan Westermann, Marie-Luise Kesting, and Tania M Lincoln. 2012. Being deluded after being excluded? How emotion regulation deficits in paranoia-prone individuals affect state paranoia during experimentally induced social stress. Behavior Therapy 43, 2 (2012), 329-340.

[25] David B Yaden, Jonathan Iwry, Kelley J Slack, Johannes C Eichstaedt, Yukun Zhao, George E Vaillant, and Andrew B Newberg. 2016. The overview effect: Awe and self-transcendent experience in space flight. Psychology of Consciousness: Theory, Research, and Practice 3, 1 (2016), 1. 